\title{
Video Quality Prediction Using a 3D Dual-Tree Complex Wavelet Structural Similarity Index
}

\author{
K. Yonis and R.M. Dansereau \\ Department of Systems and Computer Engineering, \\ Carleton University, Ottawa, Ontario, Canada \\ \{kyonis,rdanse\}@sce. carleton.ca
}

\begin{abstract}
In this paper, we test the performance of the complex wavelet structural similarity index (CW-SSIM) using the 2D dual-tree complex wavelet transform (DT-CWT). Also, we propose using a 3D DT-CWT with the $\mathbb{C W}$-SSIM algorithm, to predict the quality of digital video signals. The $2 \mathrm{D}$ algorithm was tested against the LIVE image database and has shown higher correlation with the subjective results than peak signal-to-noise ratio (PSNR), structural similarity (SSIM), and the initial steerable pyramid implementation of $\mathbb{C W}$-SSIM. The proposed 3D DT-CWT implementation of the $\mathbb{C W}$-SSIM is tested against a set of subjectively scored video sequences from the video quality experts group's (VQEG) multimedia (MM) project and gave promising results. Both implementations were validated to be good quality assessment tools to be embedded with DT- $\mathbb{C W T}$ based image and video denoising algorithms as well as DT-CWT image and video coding algorithms.
\end{abstract}

Keywords: Objective video quality assessment, dual-tree wavelet, structural similarity.

\section{Introduction}

With increasing development in video related applications, the need for a reliable method to verify and rate the quality of coded and processed video has become evermore important. As humans are often the final consumers of video information, subjective quality assessment is considered to yield the best results. However, due to the challenges in preparing subjective tests, cost, and time consumption, research efforts have developed with the goal of quantitatively predicting the quality of the final video in a way that correlates with subjective scores.

Element based objective quality assessment algorithms, such as the mean squared error (MSE) and peak signal-to-noise ratio (PSNR), have often been used to assess signal quality, due to their simplicity and their direct relation to the distortion in the signal. However, such algorithms are less meaningful when it comes to image and video signals and do not correlate well with subjective results [1, 2]. To that effect, many research efforts, including [3 7], proposed objective quality assessment algorithms that simulate human visual system (HVS) characteristics such as perceptual channels, contrast sensitivity and contrast masking.

A. Elmoataz et al. (Eds.): ICISP 2010, LNCS 6134, pp. 359-367, 2010.

(C) Springer-Verlag Berlin Heidelberg 2010 
As directly simulating the features of the HVS can be complex and leads to high processing overhead, a new way of looking at the HVS lead to a new class of objective quality algorithms. Recognizing that the HVS is more oriented towards identifying structure information in images, the structure information was used to predict the quality of images in a full reference (FR) manner, leading to the development of the structural similarity (SSIM) index and its extensions [1, 8, 9].

While having an objective video quality assessment algorithm is important, being able to embed that quality assessment as part of the processing algorithm can allow further system optimization and quality control. Unfortunately, embedding quality assessment into any processing algorithm increases the algorithm's complexity and computational overhead. To limit computational overhead, we are also motivated to develop a simple, yet effective, video quality assessment algorithm. The simplicity of the SSIM algorithm, along with its good performance, makes it a solid starting point for developing simple video assessment algorithms.

The dual-tree complex wavelet transform (DT-CWT) is a relatively new transform [10]. Nonetheless, the DT-CWT has shown good results in digital video denoising [11]. Also, the transform gave good results in video coding, without the need for a motion estimation/compensation step [12].

In this paper, we extend the complex wavelet SSIM (CW-SSIM) algorithm to use the 3D DT-CWT and use it to predict the quality of processed video signals in a FR manner. The approach will be evaluated as a stand-alone algorithm without the use of a preprocessing (registration or motion compensation) step.

The rest of this paper is organized as follows. In Sec. 2, we review the SSIM index and its complex wavelet extension $\mathbb{C W}$-SSIM. In Sec. 3, we provide a background on the dual-tree complex wavelet transform. In Sec. 4 and Sec. [5] we discuss the implementation and evaluation of the $\mathbb{C W}$-SSIM using the 2D and 3D DT-CWT respectively. Finally, conclusions are drawn in Sec. 6 .

\section{Structural Similarity Index}

Including known HVS characteristics into a video quality assessment algorithm currently results in slow and very complicated systems. A need for a simple, yet effective, algorithm emerged, where focusing on certain aspects of the HVS lead to the development of the structural similarity (SSIM) algorithm [8]. Recognizing that the HVS is more adapted to extract the viewed scene's structure information than detecting absolute differences in intensities, Wang et al. [8] developed the SSIM index to estimate the quality of an image based on structural changes between the distorted and undistorted image.

For two images $x$ and $y$, where $x$ is considered to be the perfect quality and $y$ is a distorted version of $x$, the structure information $\varsigma$ of each image is calculated by removing the luminance information $\mu$, and then normalizing by the contrast information $\sigma$ to get $\varsigma_{x}=\left(x-\mu_{x}\right) / \sigma_{x}$. Three comparison functions are derived from the extracted information of the two images; a luminance function $l(x, y)$, 
a contrast function $c(x, y)$ and a structure function $s(x, y)$. The three functions are defined and combined in [8] to give

$$
\operatorname{SSIM}(x, y)=\frac{\left(2 \mu_{x} \mu_{y}+C_{1}\right)\left(2 \sigma_{x y}+C_{2}\right)}{\left(\mu_{x}^{2}+\mu_{y}^{2}+C_{1}\right)\left(\sigma_{x}^{2}+\sigma_{y}^{2}+C_{1}\right)}
$$

where $C_{1}$ and $C_{2}$ are small positive constants used for numeric stability.

The SSIM index has shown a higher correlation with subjective scores than PSNR. Also, SSIM is not very sensitive to small variations in luminance or contrast. However, it is very sensitive to translations, rotations, and scaling due to its operation in the spatial domain.

To address these sensitivities, [1] proposed moving the SSIM algorithm from the spatial domain to the complex wavelet domain where these non-structural distortions have a smaller effect on the final quality index. This move to the complex wavelet domain made the algorithm less sensitive to small geometric distortions that are not detectable by the human eye and do not affect the perceived quality of the image.

The complex wavelet structural similarity [1] is defined in the following manner; if $c_{x}=\left\{c_{x, i} \mid i=1, \ldots, N\right\}$ and $c_{y}=\left\{c_{y, i} \mid i=1, \ldots, N\right\}$ are two sets of complex wavelet coefficients extracted from the same locations in the decomposition from the reference and distorted images, respectively, then the $\mathbb{C W}$-SSIM is given by

$$
\mathbb{C W}-\operatorname{SSIM}\left(c_{x}, c_{y}\right)=\frac{2\left|\sum_{i=1}^{N} c_{x, i} c_{y, i}^{*}\right|+K}{\sum_{i=1}^{N}\left|c_{x, i}\right|^{2}+\sum_{i=1}^{N}\left|c_{y, i}\right|^{2}+K} .
$$

The results presented in [1] for the $\mathbb{C W}$-SSIM algorithm are for a steerable pyramid wavelet transform, where (2) is applied on $7 \times 7$ coefficient blocks extracted at matching spatial locations from the same decomposition band of both the reference and distorted images. For quality evaluation, the index of each band is calculated as the average quality of all the blocks in the band and the overall quality of the image is taken as the mean of all the bands' quality values.

\section{Dual-Tree Complex Wavelet Transform}

As an alternative to the steerable pyramid wavelet transform used in [1], in this paper we propose using the dual-tree complex wavelet transform, which has been successfully used for video coding [12] and video denoising [11]. Being able to use the DT-CWT for video quality assessment could improve the coding/processing performance of these applications that use the DT-CWT.

The dual-tree complex wavelet transform (DT-CWT) is a relatively new wavelet transform [10], designed with the shortcomings of the discrete wavelet transform (DWT) in mind. Unlike the DWT, the DT-CWT is designed to be almost shift invariant with good direction selectivity 10]. The strong directional orientation of the DT-CWT comes from its ability to separate positive from negative frequencies into different bands. Similar to steerable filters, the DT-CWT 
is an overcomplete wavelet transform with a limited redundancy of $2^{d}$, where $d$ is the number of dimensions in the processed signal [10].

The DT-CWT is designed with two wavelet basis in a Daubechies-like algorithm. The first wavelet can be derived in a similar fashion to that of the DWT. The second wavelet basis $\Psi_{g}(t)$ is derived from the first basis forming a Hilbert transform pair $\Psi_{g}(t)=\mathcal{H}\left(\Psi_{h}(t)\right)$. Although both wavelet bases are real and yield their own set of coefficients bands, $\Psi_{h}(t)$ is taken as the real part of the transform and $\Psi_{g}(t)$ is treated as the complex part. With that, $\Psi(t)=\Psi_{h}(t)+i \Psi_{g}(t)$.

The 2D DT-CWT is taken as $\Psi(x, y)=\Psi(x) \Psi(y)$. With the use of the different combinations, $\Psi(x) \overline{\Psi(y)}, \Phi(x) \Psi(y), \Psi(x) \Phi(y), \Phi(x) \overline{\Psi(y)}, \Psi(x) \overline{\Phi(y)}$, where $\Phi_{h}(t)$ is the wavelet's scaling function and $\overline{\Phi(y)}$ is the conjugate of $\Phi(y)$, we can obtain 6 different orientation wavelets, each with its own unique spatial orientation. The 3D DT-CW-SSIM is defined as $\Psi(x, y, z)=\Psi(x) \Psi(y) \Psi(z)$ and the 28 different orientation wavelets are derived in a similar fashion to that of the $2 \mathrm{D}$ transform. For a detailed discussion regarding the DT-CWT, see [10, 11].

\section{Dual-Tree Complex Wavelet SSIM for Image Assessment}

In this section, we will evaluate how well the DT-CWT works for image quality assessment using $\mathbb{C W}$-SSIM. To validate the performance of this combination, which we will call the dual-tree complex wavelet SSIM (DT-CW-SSIM), we use the DT-CWT to transform both the reference and distorted images into the complex wavelet domain. This is different from what was used in testing the original $\mathbb{C W}$-SSIM implementation in [1] and what was used for the analysis in [2], which both used the steerable pyramid (SP) transform.

To predict the quality of the image using the DT-CW-SSIM algorithm, we used a 4 level 6 orientation decomposition and broke each resulting band of both the reference and test images into $7 \times 7$ overlapping blocks. The final quality index was computed as the mean of the sub-blocks' quality indices as described in [1]. We performed the image quality assessment on the entire LIVE image database [13], with results shown in Fig. 1] As seen by the scatter plots in Fig. 1. we show the quantitative measure against the difference mean opinion score (DMOS) as subjectively tested for each image. Four separate quantitative measures are shown, including PSNR in Fig. 1(a), the original spatial SSIM in Fig. 1(b), the steerable pyramid with $\mathbb{C}$-SSIM (SP-CW-SSIM) as presented in [1] in Fig. 1(c), and our use of the DT-CW-SSIMin Fig. 1(d).

As we can see from the SSIM results in Fig. 1(b), a reasonable correlation with perceptual measures is achieved. However, the SP-CW-SSIM and DT-CWSSIM results in Fig. 1(c) and Fig. 1(d) show a tighter correlation over SSIM, particularly since the images corrupted by white noise are brought in tighter with the rest of the image distortion types. From the scatter plots in Fig. 1(c) and Fig. 1(d), it is difficult to gauge what performance differences exists between SP-CW-SSIM and DT-CW-SSIM, so additional correlation tests were performed and are tabulated in Table 1. 


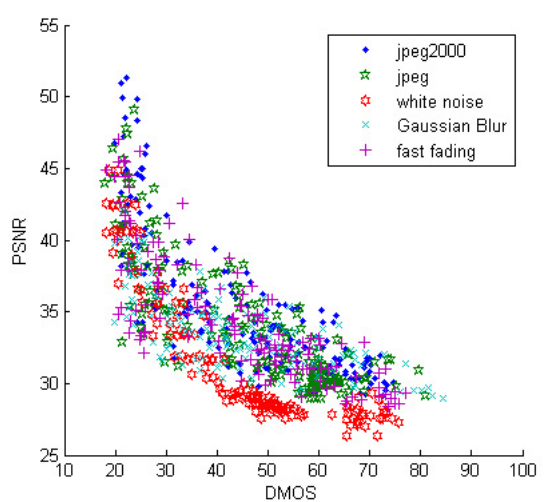

(a) PSNR

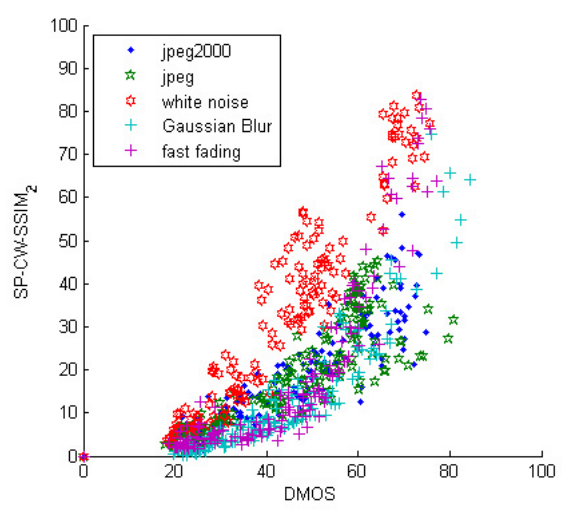

(c) PSNR

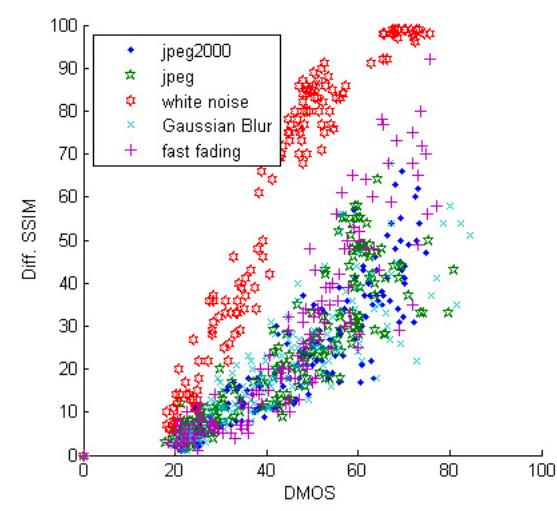

(b) SSIM

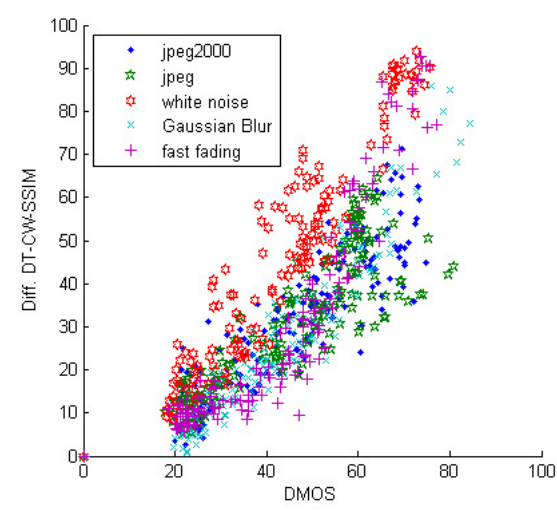

(d) SSIM

Fig. 1. Scatter plots of different quality assessment results vs. the DMOS values for all the images in the LIVE image database [13]

For each test in Table1, both the correlation coefficient (CC) and Spearman's rank order correlation coefficient (ROC) were calculated to show the algorithms' prediction accuracy and monotonicity, respectively. Another correlation coefficient was calculated $\left(\mathrm{CC}_{2}\right)$ between the DMOS values and non-linearly mapped objective results. For the non-linear mapping between the objective and subjective scores, we used the logistic function adopted in the video quality experts group (VQEG) Phase II FR-TV test [14]. It can be seen from Table1 that both $\mathbb{C W}$-SSIM algorithms outperform the PSNR and the spatial SSIM tests. Also, the results indicate that although the rank-order correlation values of both the SP-CW-SSIM and the DT-CW-SSIM are very close, the DT-CW-SSIM algorithm has a higher accuracy reflected by its correlation coefficient values. From the results in Fig. 1 and Table1, we conclude that the 2D DT-CW-SSIM provides 
Table 1. Correlation Coefficient (CC), non-linear mapped Correlation Coefficient $\left(\mathrm{CC}_{2}\right)$ and Rank Order Correlation Coefficient (ROCC) of the objective quality results vs. DMOS for all images in the LIVE image database

\begin{tabular}{c|c|c|c}
\hline Objective test & CC & $\mathrm{CC}_{2}$ & ROCC \\
\hline PSNR & 0.7883 & 0.8881 & 0.8930 \\
SSIM & 0.7506 & 0.9079 & 0.9040 \\
SP-CW-SSIM & 0.8262 & 0.9140 & 0.9334 \\
DT-CW-SSIM & 0.8866 & 0.9309 & 0.9378 \\
\hline
\end{tabular}

a slight performance gain over 2D SP-CW-SSIM. The next section explores the performance of extending the DT-CW-SSIM to a 3D implementation to include the temporal component of video.

\section{3D Dual-Tree Complex Wavelet SSIM for Video Assessment}

The good results that the 3D DT-CWT showed in video coding algorithms without the need for a motion compensation step encouraged us to implement the $\mathbb{C W}$-SSIM algorithm using the 3D DT-CWT. The motivation behind such an implementation is to have a quality assessment algorithm that is simple and can be integrated into DT-CWT based algorithms to provide a quality control step. Also, due to the DT-CWT's good directional selectivity [12] (orientation motion) and its near shift invariant design, we believe that using this transform can drastically reduce the need for computationally intensive registration steps, such as what is used in [6].

The 3D DT-CW-SSIM implementation operates on 3D blocks following the same steps as that of the original 2D implementation. To predict the quality of the video sequence, both reference and test video sequences are transformed using the 3D DT-CWT, which extracts the sequence's information into different $3 \mathrm{D}$ frequency and orientation bands. After the sequence decomposition, we break each frequency-orientation band into overlapping $3 \mathrm{D}$ blocks and calculate the CW-SSIM index for each.

Let $\left\{X=x_{i}, i=1,2, \ldots, I\right\}, I=$ number of coefficients in $X$, be the set of coefficients from an extracted 3D band's sub-block of the reference video. Also, let $\left\{Y=y_{i}, i=1,2, \ldots, I\right\}$ be the corresponding sub-block from the test sequence. Then, the sub-block's quality can be calculated directly using (2). The frequency/orientaion band's quality is the mean of all its sub-block qualities, the decomposition level's quality is the mean of all its bands' qualities, and the final video's quality index is taken as the average of the different decompositions levels' quality indices.

To test the proposed approach, we implemented the $\mathbb{C W}$-SSIM algorithm with Selesnick's implementation of the DT-CWT. To reduce the memory and 
time requirement of the algorithm, we divided each video into a number of group of pictures (GoP) with a length of 16 frames each. The algorithm was applied to each set of reference and test GoPs, decomposing each GoP into three decomposition levels with 28 orientation bands each. We discarded the low frequency approximation subbands as most of the structure information has been extracted into the detail subbands of the transform. The extracted sub-blocks from the detail bands had a fixed size of $7 \times 7$ coefficients spatially and a variable temporal depth, with its upper limit being 7 , adjusted based on the temporal depth of each decomposition band. The final video quality was computed as the average of all the GoP's quality values.

To test the proposed approach, we obtained a set of 112 subjectively scored common intermediate format (CIF) test videos. The video sequences were processed by Nortel, in a collaborative effort with the video lab at the Communications Research Center Canada (CRC), as part of the VQEG Multimedia (MM) project to evaluate objective quality assessment algorithms for CIF and QCIF video sequences. More on VQEG's multimedia project can be found in the project's Phase I report [15].

The set's test video cases were designed to reflect impairments from encoding compression and network transmission impairments (packet loss). The test video sequences used were generated from 7 reference CIF sequences, with 16 impaired sequences from each of the reference videos. The impairments in the test videos were generated through a combination of H.264 compression at bit rates of 720 , 256 , or $128 \mathrm{bps}$, frame rates of 30,20 , or $15 \mathrm{fps}$; and packet loss percentages of $0.0 \%, 0.5 \%, 0.1 \%, 0.2 \%, 0.4 \%$, or $0.8 \%$.

We used the same criteria to evaluate the video 3D DT-CW-SSIM algorithm as done with the 2D implementation. To compare the performance of the proposed approach, we implemented the video-SSIM algorithm proposed by Wang et al. in [9]. We selected the video-SSIM algorithm because it is also a video extension of the SSIM algorithm, though it only uses a 2D version of SSIM, and because the video-SSIM algorithm is reported to have good performance with the VQEG'S FRTV phase I test [9].

We have tested both implemented algorithms, video-SSIM and 3D DT-CWSSIM, against the full set of test video sequences. As shown in Table2, the results of the two video quality assessment measures indicate that the $3 \mathrm{D}$ DT-CWSSIM algorithm has a significant performance increase over the spatial videoSSIM algorithm. Although the 3D DT-CW-SSIM outperformed video-SSIM, we do see that the correlations are still rather low. Recognizing that the selected 3-D DT-CWT implementation has limitations in representing fast motion [11, 16], we ran tests on two of VQEG's MM project's sequence with low motion, CRC_SRC_bench_cif and CRC_SRC_birches1_cif, and show the results in the last row of Table 2, It seems evident that fast motion in the video can further degrade the quality of the assessments since there is a marked difference when only low motion videos are assessed. 
Table 2. Correlation coefficient $(C C)$, non-linear mapped correlation coefficient $\left(C C_{2}\right)$ and rank order correlation coefficient $(R O C C)$ of the 3D DT-CW-SSIM objective quality results vs. DMOS for VQEG's MM test video sequences

\begin{tabular}{|c|c||c|c|c|}
\hline \hline & Objective test & $C C$ & $C C_{2}$ & $R O C C$ \\
\hline \multirow{2}{*}{ all videos } & video-SSIM & 0.3776 & 0.4031 & 0.5111 \\
& 3D-DT-CW-SSIM & 0.6975 & 0.7096 & 0.7221 \\
\hline \multirow{2}{*}{ low motion video } & video-SSIM & 0.7981 & 0.8007 & 0.7598 \\
& 3D-DT-CW-SSIM & 0.8095 & 0.8416 & 0.8510 \\
\hline
\end{tabular}

\section{Conclusion}

This paper presented results of the $\mathbb{C W}$-SSIM algorithm using the $2 \mathrm{D}$ and 3D DT-CWT. The 2D implementation was evaluated against the LIVE image database. The DT-CWT implementation showed higher correlation values with subjective scores compared to PSNR, SSIM, and the original steerable pyramid implementation of the algorithm. Also, we have proposed and tested a 3D extension of the $\mathbb{C W}$-SSIM algorithm. The 3D DT-CW-SSIM implementation was tested against a set of video sequences from VQEG's MM project. The implementation showed very promising performance results, given that the algorithm is very simple and does not use any preprocessing/registration steps.

We have shown that using the DT- $\mathbb{C W T}$ with the $\mathbb{C W}$-SSIM algorithm provides a good assessment algorithm for both images and video sequences. In its current implementation, the 3D DT-CW-SSIM algorithm's performance is better for low motion video sequences; for faster motion, the performance of the 3D DT-CW-SSIM deteriorates. While not yet tested, we expect that including a rough registration step as a preprocessing stage would further enhance the performance of the 3D DT-CW-SSIM and likely allow a broader range of motion in videos for quality assessment.

Acknowledgements. The authors would like to thank Nortel and the Natural Sciences and Engineering Research Council of Canada for funding this research.

\section{References}

1. Wang, Z., Simoncelli, E.: Translation insensitive image similarity in complex wavelet domain. In: Proc. of IEEE Intern. Conf. on Acoustics, Speech and Signal Processing, March 2005, pp. 573-576 (2005)

2. Brooks, A.C., Zhao, X., Pappas, T.N.: Structural similarity quality metrics in a coding context: Exploring the space of realistic distortions. IEEE Trans. on Image Processing 17(8), 1261-1273 (2008)

3. Voran, S.: The development of objective video quality measures that emulate human perception. In: Proc. of GLOBECOM, pp. 1776-1781 (1991)

4. van den Branden Lambrecht, C., Verscheure, O.: Perceptual quality measure using a spatio-temporal model of the human visual system. In: Proc. SPIE, San Jose, CA, January 1996, vol. 2668, pp. 450-461 (1996) 
5. Winkler, S.: A perceptual distortion metric for digital color video. In: Proc. SPIE, pp. 1-4 (1999)

6. Wolf, S., Pinson, M.H.: Video quality measurement techniques. National Telecommunications and Information Administration, Report 02-392 (June 2002)

7. Yao, S., Lin, W., Ong, E., Lu, Z.: A wavelet-based visible distortion measure for video quality evaluation. In: Proc. of IEEE Intern. Conf. on Image Processing, October 2006, pp. 2937-2940 (2006)

8. Wang, Z., Bovik, A., Sheikh, H., Simoncelli, E.: Image quality assessment: From error visibility to structural similarity. IEEE Trans. on Image Processing 13(4), 600-612 (2004)

9. Wang, Z., Lu, L., Bovik, A.: Video quality assessment based on structural distortion measurement. Signal Processing: Image Communication 19(2), 121-132 (2004)

10. Selesnick, I.W., Baraniuk, R.G., Kingsbury, N.G.: The dual-tree complex wavelet transform. IEEE Signal Processing Magazine 22(6), 123-151 (2005)

11. Selesnick, I.W., Li, K.Y.: Video denoising using $2 \mathrm{~d}$ and $3 \mathrm{~d}$ dual-tree complex wavelet transforms. In: Wavelet Appl. Signal Image Proc. X, Proc. SPIE 5207, pp. 607-618 (2003)

12. Wang, B., Wang, Y., Selesnick, I., Vetro, A.: Video coding using 3d dual-tree wavelet transform. EURASIP J. on Image and Video Processing 2007(1), 15 (2007)

13. Sheikh, H.R., Wang, Z., Cormack, L., Bovik, A.C.: Live image quality assessment database release 2, http://live.ece.utexas.edu/research/quality

14. VQEG, FRTV phase II report, final report from the video quality experts group on the validation of objective models of video quality assessment, VQEG, Tech. Rep. (August 2003)

15. VQEG, "Final report from the video quality experts group on the validation of objective models of multimedia quality assessment, phase I," VQEG, Tech. Rep. (September 2008)

16. Shi, F., Selesnick, I.W.: Video denoising using oriented complex wavelet transforms. In: Proc. of IEEE Intern. Conf. on Acoustics, Speech, and Signal Processing, May 2004, vol. 2, pp. 949-952 (2004) 